\title{
Identification of Novel CDK Inhibitors by Molecular Docking and Consensus Scoring Approach
}

\author{
Satyanarayana Kotha \\ Department of Information \\ Technology \\ Sir C R Reddy college of \\ Engineering, Eluru, India
}

\author{
Yesubabu Adimulam, \\ $\mathrm{PhD}$ \\ Department of Computer \\ Science \& Engineering \\ Sir C R Reddy college of \\ Engineering, Eluru, India
}

\author{
Kiran Kumar Reddi, PhD \\ Department of Computer \\ Science \\ Krishna University \\ Machilipatnam, India
}

\begin{abstract}
Cyclin Dependent Kinases go about as potential remedial focuses in cancer disease and several efforts are under way to find out more specific, potent and selective CDK inhibitors. In this paper, reported a computational molecular docking approach to screen approved drugs from DrugBank database. Docking and scoring of all compounds was done using Molegro Virtual Docker software, evaluated the binding affinities towards CDK-2, 4, 5 and 9 enzymes 2W9F, 2UZO, $1 \mathrm{UNH}$ and 3BLR resulted in variable dock scores. The resultant top 14 hits from a dataset of 1584 approved drugs were found to be more specific towards CDK inhibition. Further, re-scoring of 14 best docked poses followed by a consensus scoring approach retrieved top hits. In this study, tested three different scoring functions such as MolDock score of Molegro software, GOLD score and AutoDock. From the analysis, it was observed that Olmesartan and Telmisartan were reported to have high binding affinities with all CDKs tested.
\end{abstract}

\section{Keywords}

Cyclin-dependent kinases, Molecular docking, Consensus scoring, Binding affinity, DrugBank database, Olmesartan, Telmisartan, Protein Data Bank.

\section{INTRODUCTION}

Cell proliferation is a significant event that takes place due to the positive and negative signals which promote cell division and suppress the process [1]. The cell cycle is governed by a family of proteins, cyclin dependent kinases (CDKs). Cyclin dependent kinases are a gathering of serine/threonine kinases which assume a pivotal part in cell cycle control [2] and are included in diverse cellular processes, in regulation of cell division (CDKs1, 2, 3, 4, 6 and 7), transcription(CDKs7, 8 and 9) or support of the structure of the cytoskeleton (CDK5) [3]. Cyclin dependent kinases control the cell cycle movment working at the transition from G2 to M, G1 to S stages, and progression through $\mathrm{S}$ stage. During the procedure, a complex set of mechanisms, for example, cyclins, phosphorylations, and endogenous CDK inhibitors at different check points are included [4]. Cell cycle progresses by the activation of Cyclin and CDK complexes [5]. Cyclins act as check points which regulate the transition from one phase of cell cycle to another where certain mitogenic signals are required for their activities and progression. Moreover, CDKs 1, 2, 4 and 5 are found to be necessary to complete a cell cycle.

CDKs require cyclin subunits for activity. Activation of CDK2 results in rotation of $\mathrm{N}$ - and C-terminal domains leading to a slight widening of ATP cleft [6]. The movement of PSTAIRE helix and Glu51 and the subsequent reorganization leads to reshaping of the phosphate-binding site [7] in case of CDK-2. During mitosis CDKs phosphorylate many distinct proteins. These CDK substrates are phosphorylated at serine or threonine deposits that are perceived by the dynamic site of the CDK protein [8]. In most cases, the target serine (S) or threonine $(\mathrm{T})$ residue is followed by a proline $(\mathrm{P})$. The typical phosphorylation succession for CDKs is $\left[\mathrm{S} / \mathrm{T}^{*}\right] \mathrm{PX}[\mathrm{K} / \mathrm{R}]$, where $\mathrm{S} / \mathrm{T}^{*}$ shows the phosphorylated serine or threonine, $\mathrm{X}$ represents to any amino acid and $K / R$ represents the basic amino acid lysine $(K)$ or arginine $(\mathrm{R})[9]$.

All CDK inhibitors concentrated so far act by rivaling ATP for binding in the CDK ATP binding pocket [10]. CDK inhibitors raduce the kinase activites of the cyclin/CDK complexs, blocking the transition from G1 to S stages [11].It has been reported that CDK4/6 inhibitors displayed promising results in the treatment of breast cancer. Selective, ATPcompetitive CDK4 inhibitors have been reported in literature [12-15] which are known to prevent phosphorylation and inactivation of $\mathrm{Rb}$ thereby inducing G1 stage leading to cell cycle arrest [16]. Success of few CDK4 inhibitors in early clinical phase trials has focused research to evaluate latephase trials against breast cancer [17]. Based on the studies that explored various CDK bound inhibitors and their role in cell cycle progression and proliferation, CDK group of enzymes are thought to act as potential therapeutic targets in several proliferative diseases [8].

From literature it was identified that several drugs are known to act as anti-cancer agents, such as chemically synthesized and evaluated drugs or those which were obtained from plant sources such as natural products. However, there is a pressing need to discover novel, more potent and efficacious compounds as anti-cancer agents. Hence, in this paper presented a novel screening approach to identify potential anti-cancer agents against cell cycle enzymes, by screening approved drugs against enzymes participating in cell cycle process using computer-aided drug design procedures.

\section{MATERIALS AND METHODS}

\subsection{Receptor X-ray Structure}

The X-ray crystal structures of cell cycle regulator proteins (CDK-2, 4, 5 and 9; 2W9F, 2UZO, 1UNH and 3BLR) were recouped from Protein Data Bank ( http://www.rcsb.org/pdb) and chosen as receptor models in virtual screening program. DrugBank database used as chemical compound library and employed three docking programs viz., Molegro Virtual Docker [18], GOLD [19] (Genetic Optimization for Ligand Docking) and AutoDock [20] for virtual ligand docking and a consensus scoring and ranking was employed to generate classes and the one with best rank was chosen. 


\subsection{Selection of Drugs from Drug Bank Database}

The Drug Bank database is an one of a kind asset of drugs with detailed data on drug and complete drug target. The database contains nearly 7740 drug entries including 1584 FDA-approved small molecule drugs, 157 FDA-approved biotech (protein/peptide) drugs, and $>6000$ experimental drugs [21]. In the present study, 1584 approved drugs were selected for analysis.

\subsection{Molegro Virtual Docker}

Molegro Virtual Docker (MVD) is an integrated platform for predicting protein - ligand interactions. All default options including preparation of the molecules to determination of the potential binding sites of the target protein, and prediction of the binding modes of the ligands were employed. The MVD has been shown to yield higher docking accuracy than other state-of-the-art docking products [18].

\subsection{Gold}

The GOLD program uses a genetic algorithm (GA) and the binding site was defined as a spherical region which encompasses all protein atoms within $5.0 \AA$ of each crystallographic ligand atom. Default settings were used for all calculations. For each of the 10 independent GA runs, a maximum number of $10000 \mathrm{GA}$ operations were performed on a single population of 50 individuals. Operator weights for crossover, mutation, and migration were set to 100, 100 and 0 .

To further speed up the calculation, the GA docking was stopped when the top three solutions were within $1.5 \mathrm{~A}^{\circ}$ RMSD (Root Mean Square Deviation) of each other.

\subsection{AutoDock}

AutoDock requires receptor and ligand coordinates in MOL2 or PDB format. Nonpolar hydrogen atoms were removed from the receptor file and their partial charges were added to the corresponding carbon atoms. All docking runs were performed using the Lamarckian genetic algorithm and the best value was reported in $\mathrm{kcal} / \mathrm{mol}$. The standard docking protocol consisted of 10 independent runs per ligand, using an initial population of 50 randomly placed individuals, a maximum number of $2.5 \times 10^{5}$ energy evaluations, a mutation rate of 0.02 , a crossover rate of 0.80 , and an elitism value of 1 . The probability of performing a local search on an individual in the population was 0.06 , using a maximum of 300 iterations per local search. Results differing by less than $2 \AA$ RMSD from each other were clustered together and represented as best docking energy [20].

\subsection{Molecule Preparations}

Although DrugBank database provides ligands in 3-D formats, an energy minimization routine was performed to generate three dimensional structures of all the molecules using corina make 3D option, derived charges and the geometries were optimized using cosmic module of Tsar Software. Water molecules were discarded from the PDB file, and missing side chains were reconstructed using WHAT-IF web

interface

(http://swift.cmbi.ru.nl/servers/html/index.html). Hydrogens were added and then the structure was converted to mol2 format using Mercury (http://www.ccdc.cam.ac.uk/products/mercury) (v. 1.4.2; Cambridge Crystallographic Data Centre (CCDC)).

\subsection{Consensus Scoring and Ranking}

Docking programs predict binding modes and energies of protein-ligand complex structures with reasonable accuracy and speed. Most of the docking programs are able to predict the nearer binding mode of a ligand, however, scoring functions play major role to differentiate correct poses from incorrect ones. As docking and scoring play important roles in drug design, it has been pointed out that the major weakness of docking programs lies in scoring functions. However, combinations of various scoring functions would reduce the errors in single scoring schemes and improve the probability of identifying true hits [22]. Thus, it has been demonstrated that consensus scoring was generally more effective than single scoring for molecular docking [23] and represented an effective way in getting improved hit rates in various virtual database screening studies [24].

In this study, tested three different scoring functions such as MolDock score of Molegro software, GOLD score implemented in GOLD 3.1, and Free energy score of AutoDock. Docking program Molegro Virtual Docker was used to dock DrugBank compounds and the generated ensemble of docked conformations were scored and applied to generate classes followed by ranking the best conformations. The best conformations of each ligand that were clustered using complete linkage analysis are saved in mol 2 formats. These files are used to apply the remaining scoring functions. During ranking, signs of some scoring functions are changed to make certain that a lower score always indicates a higher affinity.

\section{RESULTS AND DISCUSSION}

The docking protocol was validated before screening DrugBank database. 2 UZO protein bound ligand was docked into the binding pockets of all CDKs to obtain the docked pose and the RMSD (Root Mean Square Deviation) of all atoms between these two conformations is $<2.0^{\circ} \mathrm{A}$ indicating that the parameters for docking simulation are reasonable in reproducing the X-ray crystal structure. Therefore, DrugBank database was screened for all approved drugs, which were docked into each of the protein structures using default parameters of Molegro virtual docker. Table 1 shows the binding affinities and RMSD values of cell cycle proteins studied.The rationale behind selecting $\mathrm{CDKs}$ as potential cancer targets is based on published literature on these enzymes. Of all the cancer causing or participating proteins/enzymes such as CDKs, apoptotic proteins and others, it has been reported that the crucial phases of cell cycle can be arrested if any one of the CDKs are blocked in a way to reduce cell proliferation. Hence, it has been postulated by many authors that targeting CDKs would provide a higher chance or rate of inhibiting cell cycle process. 
Table1: Mol dock scores of cell cycle regulator proteins

\begin{tabular}{|c|c|c|c|c|c|c|c|}
\hline \multirow{2}{*}{ S.NO } & \multirow{2}{*}{ PDB ID } & \multicolumn{3}{|c|}{ Mol Dock Score(kcal/mol) } & \multirow{2}{*}{$\begin{array}{l}\text { Average } \\
\text { mol dock } \\
\text { score } \\
\text { (kcal/mol) }\end{array}$} & \multirow{2}{*}{$\begin{array}{l}\text { Average } \\
\text { RMSD } \\
\left({ }^{\circ} \mathbf{A}\right)\end{array}$} & \multirow{2}{*}{ Value } \\
\hline & & Run-1 & Run-2 & Run-3 & & & \\
\hline 1 & 2W9F & 145.5 & 147.78 & 146.3 & 146.51 & 0.67758 & \\
\hline 2 & $2 \mathrm{UZO}$ & 126.54 & 123.25 & 125.63 & 125.15 & 1.06333 & \\
\hline 3 & $1 \mathrm{UNH}$ & 113.91 & 111.66 & 114 & 133.19 & 0.11708 & \\
\hline 4 & 3BLR & 114.24 & 112.32 & 114.63 & 114.06 & 0.17279 & \\
\hline
\end{tabular}

Docking of all 1584 approved drugs from DrugBank was carried out to evaluate best conformer based on the lowest docked energy $(\mathrm{kcal} / \mathrm{mol})$, in other words, it should possess highest affinity towards the binding site. Moreover, the virtual screening technique employed in this work recognized entirely diverse, yet specific drugs that bind in a comparable manner as seen with ATP binding in CDKs. Therefore, in the first step, virtual screening based on docking and scoring of DrugBank compounds resulted in few hits with dock scores reaching more than $200 \mathrm{kcal} / \mathrm{mol}$. The result is given in Table- 2 .

Table 2: Docking results of MVD

\begin{tabular}{lllll}
\hline Drug Name & $\begin{array}{l}\text { 2W9F dock } \\
\text { score } \\
(\mathbf{k c a l} / \mathbf{m o l})\end{array}$ & $\begin{array}{l}\text { 2UZO dock score } \\
(\mathbf{k c a l} / \mathbf{m o l})\end{array}$ & $\begin{array}{l}\text { 1UNH dock score } \\
(\mathbf{k c a l} / \mathbf{m o l})\end{array}$ & $\begin{array}{l}\text { 3BLR dock score } \\
\text { (kcal/mol) }\end{array}$ \\
\hline Pentagastrin & 158.351 & 149.198 & 145.323 & 156.789 \\
Olmesartan & 208.296 & 198.472 & 197.894 & 187.035 \\
Teniposide & 174.5 & 164.784 & 178.286 & 166.59 \\
Verteporfin & 200.02 & 223.223 & 185.378 & 213.67 \\
Montelukast & 175.412 & 167.435 & 155.934 & 163.284 \\
candoxatril & 165.536 & 154.91 & 155.38 & 169.491 \\
Pemetrexed & 162.825 & 138.96 & 159.865 & 148.055 \\
Losartan & 168.644 & 163.567 & 161.244 & 168.133 \\
Candesartan & 170.916 & 166.307 & 160.613 & 167.591 \\
Eprosartan & 181.317 & 167.877 & 161.587 & 177.815 \\
Tiagabine & 171.856 & 138.006 & 130.721 & 136.154 \\
Repoglinide & 167.581 & 150.842 & 142.284 & 138.795 \\
Telmisartan & 175.608 & 187.981 & 166.982 & 189.228 \\
Atorvastatin & 183.618 & 189.203 & 183.401 & 191.542 \\
\hline & & & & \\
\hline
\end{tabular}

In the next step, re-scoring docking poses with independent functions was another valuable approach which gained prominence in recent studies [25].Therefore, re-scoring of best docked poses from MVD was done using GOLD and AutoDock. Results are presented in Table3.Further, a consensus scoring approach was implemented to evaluate best compounds from a set of 14 finalized drugs, which showed probable best docked poses against CDKs. Consensus scoring approaches combining multiple scoring functions were shown to work better in many [26]. Hence, the
GOLD score, Molegro Score and AutoDock was applied to reevaluate the 14 docked poses. Ranking was done individually by equally splitting the dock scores into three classes using Tsar Software (www.accelrys.com). The compounds in Class3 represent the highest class or top rank. Summation of all ranks (rank-sum) was considered as best technique rather taking average values [27]. The advantage of a sum over an average was that the contribution from the rank for each individual score can more easily be split out for illustrative purposes in the former instance [27]. The details are given in table-4. 
Table 3: Dock scores comparison of top best compounds that exhibited high affinities with MVD.

\begin{tabular}{lllllllll}
\hline \multirow{2}{*}{ Drug Name } & \multicolumn{7}{c}{ GOLD $(\mathrm{kcal} / \mathrm{mol})$} & \multicolumn{7}{c}{ AutoDock (kcal/mol) } \\
\cline { 2 - 8 } & 2W9F & 2UZO & 1UNH & 3BLR & 2W9F & 2UZO & 1UNH & 3BLR \\
\hline Pentagastrin & 44.53 & 56.63 & 51.11 & 66.81 & 5.9 & 7.8 & 8 & 3.9 \\
Olmesartan & 60.26 & 63.88 & 54.01 & 60.03 & 8.5 & 8.7 & 7.8 & 5.7 \\
Teniposide & 33.31 & 42.63 & 38.35 & 9.72 & 7.3 & 8.7 & 8.6 & 5.2 \\
Verteporfin & 13.14 & 63.98 & 46.00 & 51.72 & 6.4 & 9.9 & 8.1 & 5.1 \\
Montelukast & 54.34 & 59.51 & 47.28 & 59.07 & 7.9 & 8.7 & 9.3 & 4.7 \\
candoxatril & 25.19 & 51.46 & 36.56 & 47.53 & 7.8 & 9.2 & 8.7 & 5 \\
Pemetrexed & 74.72 & 70.19 & 53.9 & 71 & 9 & 9.3 & 8.8 & 5.9 \\
Losartan & 59.11 & 64.09 & 48.68 & 72.82 & 7.4 & 9.1 & 8.2 & 8.6 \\
Candesartan & 53.51 & 64.83 & 49.45 & 67.02 & 7.6 & 8.8 & 8.8 & 8.5 \\
Eprosartan & 66.54 & 57.87 & 52.69 & 67.1 & 7.3 & 8.1 & 7.8 & 8.8 \\
Tiagabine & 57.11 & 49.58 & 39.72 & 59.06 & 7.2 & 7.3 & 7.7 & 7.4 \\
\hline
\end{tabular}

Table 4: Ranks of scores obtained in Molegro, GOLD, AutoDock for CDKs(Top three best scores are underlined).

\begin{tabular}{|c|c|c|c|c|c|c|c|c|c|c|c|c|c|c|c|c|}
\hline \multirow[t]{2}{*}{ Drug Name } & M & G & $\mathbf{A}$ & \multirow[t]{2}{*}{$\begin{array}{l}\text { Rank } \\
\text { Sum }\end{array}$} & M & G & $\mathbf{A}$ & \multirow[t]{2}{*}{$\begin{array}{c}\text { Rank } \\
\text { Sum }\end{array}$} & $\mathbf{M}$ & G & $\mathbf{A}$ & $\begin{array}{l}\text { Rank } \\
\text { sum }\end{array}$ & $\mathbf{M}$ & G & A & \multirow[t]{2}{*}{$\begin{array}{l}\text { Rank } \\
\text { sum }\end{array}$} \\
\hline & \multicolumn{3}{|c|}{$2 W 9 F$} & & \multicolumn{3}{|c|}{$2 \mathrm{UZO}$} & & \multicolumn{4}{|c|}{ 1UNH } & \multicolumn{3}{|c|}{ 3BLR } & \\
\hline Pentagastrin & 1 & 2 & 1 & 4 & 1 & 2 & 1 & 4 & 1 & 3 & 1 & 5 & 1 & 3 & 1 & 5 \\
\hline Olmesartan & 3 & 3 & 3 & $\underline{9}$ & 3 & 3 & 2 & $\underline{8}$ & 3 & 3 & 1 & $\underline{7}$ & 2 & 3 & 1 & 6 \\
\hline Teniposide & 1 & 2 & 2 & 5 & 1 & 1 & 2 & 4 & 3 & 1 & 2 & 6 & 2 & 1 & 1 & 4 \\
\hline Verteporfin & 3 & 1 & 1 & 5 & 3 & 3 & 3 & $\underline{9}$ & 3 & 2 & 1 & 6 & 3 & 2 & 1 & 6 \\
\hline Montelukast & 2 & 3 & 2 & 7 & 2 & 2 & 2 & 6 & 2 & 2 & 2 & 6 & 2 & 3 & 1 & 6 \\
\hline candoxatril & 1 & 1 & 2 & 4 & 1 & 2 & 2 & 5 & 2 & 1 & 2 & 5 & 2 & 2 & 1 & 5 \\
\hline Pemetrexed & 1 & 3 & 3 & 7 & 1 & 3 & 2 & 6 & 2 & 3 & 2 & 7 & 1 & 3 & 1 & 5 \\
\hline Losartan & 1 & 3 & 2 & 6 & 1 & 3 & 2 & 6 & 2 & 3 & 1 & 6 & 2 & 3 & 3 & 8 \\
\hline Candesartan & 1 & 2 & 2 & 5 & 1 & 3 & 2 & 6 & 2 & 3 & 2 & $\underline{7}$ & 2 & 3 & 3 & $\underline{8}$ \\
\hline Eprosartan & 2 & 3 & 2 & $\underline{7}$ & 2 & 2 & 1 & 5 & 2 & 3 & 1 & 6 & 2 & 3 & 3 & $\underline{8}$ \\
\hline Tiagabine & 1 & 3 & 2 & 6 & 1 & 1 & 1 & 3 & 1 & 1 & 1 & 3 & 1 & 3 & 2 & 6 \\
\hline Repoglinide & 1 & 1 & 1 & 3 & 1 & 1 & 1 & 3 & 1 & 1 & 2 & 4 & 1 & 2 & 3 & 6 \\
\hline Telmisartan & 2 & 3 & 3 & $\underline{8}$ & 2 & 3 & 3 & $\underline{8}$ & 2 & 3 & 3 & $\underline{8}$ & 3 & 3 & 3 & $\underline{9}$ \\
\hline Atorvastatin & 2 & 2 & 1 & 5 & 2 & 1 & 2 & 5 & 3 & 1 & 1 & 5 & 3 & 2 & 3 & 8 \\
\hline
\end{tabular}

M: Molegro Virtual Docker; G: GOLD; A: AutoDock

The best three drugs which reported to exhibit high binding affinity against all targets are considered and they are identified as:

- 2W9F:Olmesartan,Telmisartan,Eprosartan

- 2UZO: Olmesartan, Telmisartan, Verteporfin

- $\quad$ 1UNH: Olmesartan, Telmisartan, Candesartan

- 3BLR: Eprosartan, Telmisartan, Candesartan.

From the above data, it can be emphasized that Olmesartan and Telmisartan (Figure 1) would act as inhibitors against cell cycle proteins. Olmesartan, an angiotensin II receptor antagonist, chemically is (5-methyl-2-oxo- $2 \mathrm{H}-1,3$-dioxol-4yl)methyl4-(2-hydroxypropan-2-yl)-2-propyl-1-(\{4-[2-(2H1,2,3,4-tetrazol-5-yl)phenyl]phenyl \}methyl) -1H-imidazole-5carboxylate, used for the treatment of high blood pressure. Considering the imidazole ring moiety at the centre, with tetrazole and dioxolyl groups as side chains on either arm of the structure, this scaffold should be studied in much detail owing to the interactions it makes with active site amino acid residues of tyrosine kinase enzymes participating in cancer pathways. 

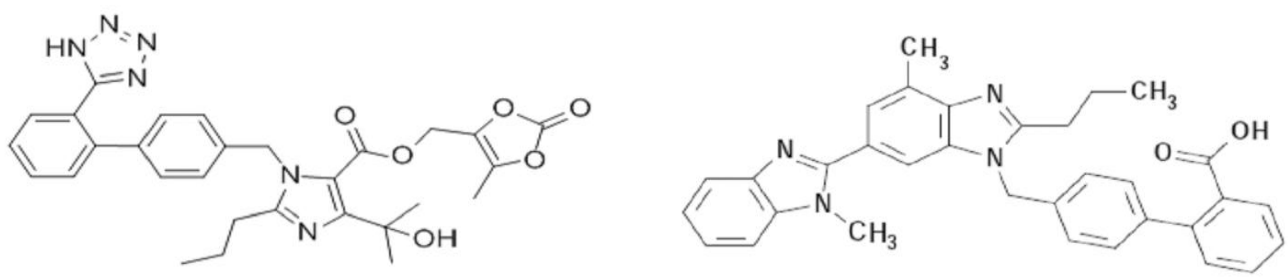

Figure 1. 2-dimensional structures of Olmesartan and Telmisartan

On the other hand, Telmisartan, an approved angiotensin II receptor antagonist has chemical structure, 2-(4-\{[4-Methyl-6(1-methyl-1H-1,3-benzodiazol-2-yl)-2-propyl-1H-1,3-

benzodiazol-1-yl]methyl phenyl)benzoic acid. This chemical has two benzodiazoles and benzoic acid moieties making it more hydrophobic groups amenable to bind with hydrophobic amino acid residues of active site region of proteins. Hence, it would like to explore the characteristic features of these two compounds and screen proteins that are reported to be active in various cancer diseases. Moreover, experimental analysis involving cytotoxicity studies would shed some light in studying the capability of these drugs to act as possible anticancer agents.

\section{CONCLUSION}

The analysis reported in this paper identified a unique method of choice to screen DrugBank compounds which resulted in diverse, novel approved drugs as possible anti-cancer agents. The computer-aided drug design protocol implemented would be advantageous in exploring novel drugs that are identified using consensus scoring and ranking techniques, on one hand and they might also provide a new scaffold for further design and development of novel CDK inhibitors. Further, work is in progress to explore computational analysis on analogs of olmesartan and telmisartan to study the possible enhanced pharmacophoric features of these compounds.

\section{REFERENCES}

[1] Brun, V., Legraverend, M., Grierson, D. S. 2001. Cyclindependent kinase (CDK) inhibitors: development of a general strategy for the construction of 2,6,9trisubstituted purine libraries. Part 1 Tetrahedron Lett. Vol. 42: 8161-8164.Ding, W. and Marchionini, G. 1997 A Study on Video Browsing Strategies. Technical Report. University of Maryland at College Park.

[2] Fischer, P. M., Endicott, J., Meijer, L. 2003. Cyclindependent kinase inhibitors. Prog Cell Cycle Re. Vol.5: 235-248.

[3] Schang, L. M. 2002. Cyclin-dependent kinases as cellular targets for antiviral drugs. J Antimicrob Chemother. Vol. 50: 779-792.Sannella, M. J. 1994 Constraint Satisfaction and Debugging for Interactive User Interfaces. Doctoral Thesis. UMI Order Number: UMI Order No. GAX9509398., University of Washington.

[4] Sausville, E. A., Johnson, J., Alley, M., Zaharevitz, D., Senderowicz, A. M. 2000. Inhibition of CDKs as a therapeutic modality. Ann N Y Acad Sci. Vol. 910: 207221.Brown, L. D., Hua, H., and Gao, C. 2003. A widget framework for augmented interaction in SCAPE.

[5] Ekholm, S. V., Reed, S. I. 2000. Regulation of G(1) cyclin-dependent kinases in the mammalian cell cycle. Curr Opin Cell Biol. Vol. 12: 676-684
[6] Davies, T.G., Pratt, D.J., Endicott, J.A., Johnson, L.N., Noble, M.E. 2002. Structure based design of cyclindependent kinase inhibitors. Pharmacol The., Vol. 93:125-133.

[7] Noble, M. E., Endicott, J. A. 1999. Chemical inhibitors of cyclin-dependent kinases: insights into design from Xray crystallographic studies. Pharmacol Ther. Vol.82: 269-278.

[8] Davies, T. G., Tunnah, P., Meijer, L., Marko, D., Eisenbrand, G., Endicott, J. A., Noble, M. E. 2001. Inhibitor binding to active and inactive CDK2: the crystal structure of CDK2-cyclin A/indirubin-5-sulphonate. Structure. Vol. 9: 389-397.

[9] DeBondt, H. L., Rosenblatt, J., Jancarik, J., Jones, H. D., Morgan, D. O., Kim, S. H. 1993. Crystal structure of cyclin-dependent kinase 2. Nature. Vol. 363:595-602.

[10] Noble, M. E., Endicott, J. A., Johnson, L. N. 2004. Protein kinase inhibitors: insights into drug design from structure. Science. Vol. 303: 1800-1806.

[11] Sherr, C. J. 2000. The Pezcoller lecture: cancer cell cycles revisited. Cancer Res. Vol. 60: 3689-3695.

[12] Takao Horiuchi., Jun Chiba., Kouichi Uoto., Tsunehiko Soga. 2009. Discovery of novel thieno[2,3-d]pyrimidin4-yl hydrazone-based inhibitors of Cyclin D1-CDK4: Synthesis, biological evaluation, and structure-activity relationships. Bioorganic \& Medicinal Chemistry Letters, Vol.19: 305-308.

[13] Concha Sanchez-Martinez., Chuan Shih., Margaret, M. Faul., Guoxin Zhu., Michael Paal., Carmen Somoza et al. 2003. Aryl[a]pyrrolo[3,4-c]carbazoles as selective cyclin D1-CDK4 inhibitors. Bioorganic\&Medicinal Chemistry Letters. Vol.13: 3835-3839.

[14] Thomas, A. Engler., Kelly Furness., Sushant Malhotra., Concha Sanchez-Martinez., Chuan Shih., Walter Xie., Guoxin Zhu et al. 2003. Novel potent and selective cyclin D1/CDK4 inhibitors:indolo[6,7-a]pyrrolo[3,4c]carbazoles .Bioorganic \& Medicinal Chemistry Letters, Vol.13: 2261-2267.

[15] Kirk, L., Stevens, Michael J. Reno., Jennifer B. Alberti., Daniel J. Price., Laurie S. Kane-Carson., Victoria B. Knick et al. 2008. Synthesis and evaluation of pyrazolo[1,5- b]pyridazines as selective cyclin dependent kinase inhibitors. Bioorganic \& Medicinal Chemistry Letters, Vol.18: 5758-5762.

[16] Choi, YJ., Anders, L. 2014. 'Signaling through cyclin Ddependent kinases. Oncogene. Vol.33: 1890-1903. 
[17] Shapiro, GI. 2006. Cyclin-dependent kinase pathways as targets for cancer treatment. J Clin Oncol. Vol.24: 17701783.

[18] Rene'Thomsen and Mikael H.Christensen. 2006. MolDock: A New Technique for High-Accuracy Molecular Docking. J. Med. Chem. Vol.49: 3315-3321.

[19] Jones, G., Willett, P., Glen, R.C., Leach, A.R., Taylor, R. 1997. Development and validation of a genetic algorithm for flexible docking. J Mol Biol. Vol. 267: 727-748.

[20] Garrett M Morris., David S Goodsell., Robert S Halliday., Ruth Huey., William E Hart., Richard K Belew., Arthur J Olson. 1998. Automated docking using a Lamarckian genetic algorithm and an empirical binding free energy function. Journal of computational chemistry. Vol.19:1639-1662.

[21] Law, V., Knox, C., Djoumbou, Y., Jewison, T., Guo, AC., Liu, Y., Maciejewski, A., Arndt, D., Wilson, M., Neveu, V., Tang, A., Gabriel, G., Ly, C., Adamjee, S., Dame, ZT., Han, B., Zhou, Y., Wishart, DS. 2014. DrugBank 4.0: shedding new light on drug metabolism. Nucleic Acids Res. Vol.42:1091-1097.

[22] Kitchen, D.B., Decornez, H., Furr, J.R., Bajorath, J. 2004. Docking and scoring in virtual screening for drug discovery: methods and applications. Nat Rev Drug Discov. Vol.3: 935-949.

[23] Wang, R., Lu, Y., Wang, S. 2003. Comparative evaluation of 11 scoring functions for molecular docking. J Med Chem. Vol.46: 2287-2303.

[24] Charifson, P.S., Corkery, J.J., Murcko, M.A., Walters, W.P. 1999. Consensus scoring: A method for obtaining improved hit rates from docking databases of threedimensional structures into proteins. J Med Chem. Vol. 42:5100-5109.

[25] Stahl, M., Bohm, H.J. 1998. Development of filter functions for protein-ligand docking. J Mol Graph Model. Vol.16:121-132.

[26] Wang, R., Lu, Y., Fang, X., Wang, S. 2004. An extensive test of 14 scoring functions using the PDB bind refined set of 800 protein-ligand complexes. J Chem Inf Model. Vol.44:2114-2125.

[27] Clark, R.D., Strizhev, A., Leonard, J.M., Blake, J.F., Matthew, J.B. 2002. Consensus scoring for ligand/protein interactions. J Mol Graph Model. Vol. 20: 281-295. 